\title{
Genetic dissection of autoimmune type I diabetes in the BB rat
}

H. J. Jacob, A. Pettersson, D. Wilson, Y. Mao, Å. Lermark \& E. S. Lander

Nature Genetics 2, 56-60 (1992)

Originally we reported that $I d d m l$ is responsible for causing lymphopenia and is tightly linked to the $N p y$ locus $(0.7 \mathrm{cM}$ from $N p y$ in the direction of $I g k$ ) on rat chromosome 4, the exact position based on three recombinants out of 429 animals. Subsequently, we have determined that the three apparent recombinants were due to sample and phenotype errors: there were no actual recombinants between $N p y$ and $I d d m I$. Studies of more than 500 additional animals have shown that $I d d m 1$ still maps very close to $N p y$, but towards the locus D4Mit6 (see Fig. 1). These findings do not affect the conclusions of the paper, apart from repositioning $I d d m I$ by approximately $1.5 \mathrm{cM}$.

\section{Automated construction of genetic linkage maps using an expert system (MultiMap): a human genome linkage map}

T. Cox Matise, M. Perlin \& A. Chakravarti

Nature Genetics 6, 384-390 (1994)

Information about MultiMap can be obtained from the following revised e-mail addresses: aravinda@chimera.gene.cwru.edu or tara@genomel.hgen.pitt.edu

\section{erratum}

\section{A null mutation in the human CNTF gene is not causally related to neurological diseases}

Ryosuke Takahashi, Hidehiro Yokoji, Hidemi Misawa, Michiyuki Hayashi, Jianguo Hu \& Takeo Deguchi

Nature Genetics 7, 79-84 (1994)

Table 1 was inadvertantly omitted from the final layout of this paper. Table 1 appears below.

Table 1 Distribution of CNTF genotypes in healthy subjects and patients with neurological diseases

Number of subjects (\%) Genotype

Subjects

Healthy volunteer

ALS

Alzheimer disease

Parkinson disease

Miscellaneous disease

Total

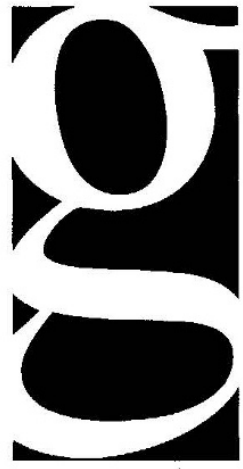

nature genetics

1234 National

Press Building

529 14th St NW

Washington DC 20045

voice $\mathrm{ph}$ :

202-626-2513

fax ph:

202-628-1609

\section{GUIDE TO AUTHORS}

NATURE GENETICS is an international monthly journal publishing important advances in all fields affecting human genetics and the Genome Project, including the identification and characterization of genes and gene products involved in hereditary disease, genetic diagnosis and therapy, molecular analysis and mapping of the genome (in all organisms), animal models, cancer and developmental genetics.

Manuscripts are selected for publication according to editorial assessment of their general interest and suitability and reports from independent referees. Receipt of all manuscripts will be acknowledged but those that are not suitable for review will be returned without delay. Contributors are welcome to suggest potential reviewers as well as informing the Editor of potential conflicts of interest. We urge clear writing to enhance the accessibility of manuscripts for readers not directly familiar with the field under investigation.

Following acceptance of their paper, contributors will receive galley proofs within a few weeks. Some editing is likely in order to enhance clarity and consistency. Contributors will receive a reprint order form with their proofs. Nature-Genetics will not exact page charges.

\section{Format of Articles}

Manuscripts should generally follow the style and format of an article in Nature, with a Summary, Introduction, Results and Discussion. Note that their is a separate Methodology section at the end of the text and we include full titles of the papers in the references. Text should be between 1,000 and 3,000 words in length.

TITLES should be simple and concise.

SUMMARY. A brief, accessible summary of no more than 100 words should explain the goals, results and chief conclusions of the work.

RESULTS should include short cross-headings to define the main aspects of the study. We request that authors deposit sequence data in the databases, and provide an accession number in the paper.

METHODOLOGY will be a separate section placed at the end of the text, before the references.

REFERENCES are to be numbered sequentially as they appear in the text, followed by those in the figure legends and tables. Do not include any annotation. Full titles of papers are required. All authors should be listed unless there are six or more, in which case 'et al.' should be substituted. First and last page numbers in full must be included; references to books should include publisher, place and date. The list should include only papers published or in press; papers submitted or in preparation and personal communications should be cited in the text.

FIGURES. Original artwork should be submitted with the manuscript. Oversized artwork should be avoided.

\section{ACKNOWLEDGEMENTS should be brief.}

SUBMISSION. Manuscripts should be sent to the Editor, NATURE GENETICS, 1234 National Press Building, 529 14th St. NW, Washington DC 20045, USA. Please provide current fax and phone numbers of the corresponding author on all submissions.Manuscripts should be typed, double-spaced, on one side of the paper only. An original and three copies are required, each accompanied by artwork, together with a computer diskette. Colour prints will be partly paid for by authors unless otherwise agreed.

ON DISKETTE. Please note that we set all page proofs directly from computer discs provided by the authors. Any common Macintosh or PC word-processing package is compatible and preferable to a text/ASCII file. (N.B. Manuscripts written in WordStar cannot be processed.) 